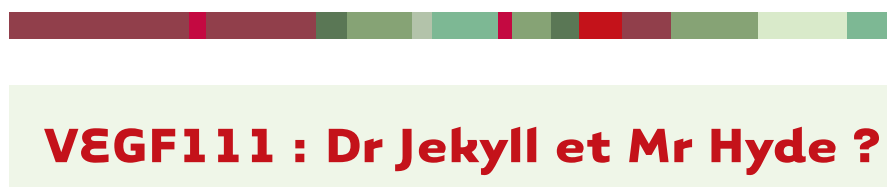

Charles A. Lambert, Pierre Mineur, Betty V. Nusgens
Laboratoire de Biologie

des Tissus Conjonctifs, GIGA-Cancer,

Tour de Pathologie B23/3, Université de Liège,

4000 Liège, Belgique.

c.lambert@ulg.ac.be

pierre.mineur@ulg.ac.be

betty.nusgens@ulg.ac.be

Enfin il est résistant à la dégradation par la plasmine et les fluides biologiques riches en protéases comme les exsudats d'ulcères. Les cellules humaines exprimant le VEGFl65, le VEGF121 ou le VEGFlll par recombinaison ont été injectées à des souris immunodéficientes. Si les cellules exprimant le VEGF165, et dans une moindre mesure celles exprimant le VEGFl21, ont produit des tumeurs vascularisées, les cellules exprimant le VEGFlll produisent des tumeurs peu vascularisées, mais dont le tissu environnant est irrigué par un réseau dense de capillaires.

\section{En cas de coupure, composer le 111} Le VEGFlll pourrait remplacer avantageusement les autres variants dans le traitement de diverses pathologies. C'est particulièrement vrai pour les ulcères veineux, l'infarctus du myocarde et l'accident vasculaire cérébral. Dans le premier cas, l'environnement de la plaie est riche en plasmine et en métalloprotéases matricielles et dégrade le VEGFl65 [5]. Un VEGF165 muté au site de clivage par la plasmine accroît la stabilité des structures vasculaires durant la cicatrisation des plaies chroniques chez les lulaire, alors que le variant 121 est actif et librement diffusible. Le variant 165 a des propriétés de liaison intermédiaires. Un variant utilisant un exon alternatif $8 b$ présente des propriétés anti-angiogènes. L'exon 5 code pour les sites principaux de clivage par la plasmine et les métalloprotéases matricielles ([2, 3] et IruelaArispe, communication personnelle).

\title{
Le VEGF111
}

Nous avons récemment cloné un nouveau variant d'épissage alternatif du VEGF,

Si notre ADN contient quelques 25000 gènes, nos cellules expriment collectivement plus de 100000 protéines de séquences différentes. La détermination de la nature et de la fonction de l'ensemble de ces protéines constitue un des défis de l'ère post-génomique. Cette complexification du répertoire protéique par rapport au répertoire génomique est rendue possible grâce notamment au mécanisme d'épissage alternatif des pré-ARN messagers en ARN messagers. Ce processus concerne plus de la moitié de nos gènes. II permet la synthèse de protéines différentes (variants) à partir d'un gène donné grâce à l'exclusion d'exons ou parties d'exons, ou la rétention d'introns ou parties d'introns, dans la séquence de l'ARN messager. Dans un certain nombre de cas, les variants produits par l'épissage alternatif d'un gène ont des propriétés différentes, voir antagonistes. 
souris diabétiques [6]. Sa résistance au clivage par des exsudats d'ulcères fait du VEGFlll une alternative séduisante, potentiellement plus efficace que le VEGF165 muté en raison de l'élimination du site de clivage par les métalloprotéases matricielles. Cette hypothèse est en cours d'étude dans notre laboratoire. Dans les deux autres cas, celui des maladies ischémiques, nombre de patients arrivant dans les services d'urgences reçoivent une injection d'activateur tissulaire du plasminogène, afin de transformer le plasminogène en plasmine capable de lyser le thrombus responsable de l'occlusion artérielle causative de la pathologie. Des travaux préliminaires suggèrent que le VEGFlll, et dans une moindre mesure le VEGFl65, réduisent le déficit de perfusion cardiaque consécutif à la ligature de l'artère coronaire gauche descendante, l'hypertrophie réactionnelle à l'ischémie et le remodelage du ventricule gauche chez la souris (Marie Delgaudine, Pierre Mineur et Charles Lambert). L'avantage du VEGFlll par rapport au VEGFl65 est faible, mais pourrait devenir significatif si ce dernier est dégradé lors de l'injection d'activateur du plasminogène aux animaux.

À l'inverse, l'utilisation de thérapies anti-angiogènes pour combattre le cancer a été proposée dès 1971 par Judah Folkman (1933-2008) [7]. Quelque trente ans plus tard, un anticorps humanisé capable de bloquer le VEGF (bevacizumab, Avastin ${ }^{\circledR}$, Genentech, États-Unis) est le premier agent antiangiogène accepté aux États-Unis par la Food and Drug Administration pour le traitement de cancers colorectaux en adjonction à la chimiothérapie [8]. Certains effets secondaires, notamment des saignements, sont cependant à déplorer. L'expression de VEGFlll par des cellules tumorales humaines transplantées chez la souris, induite par

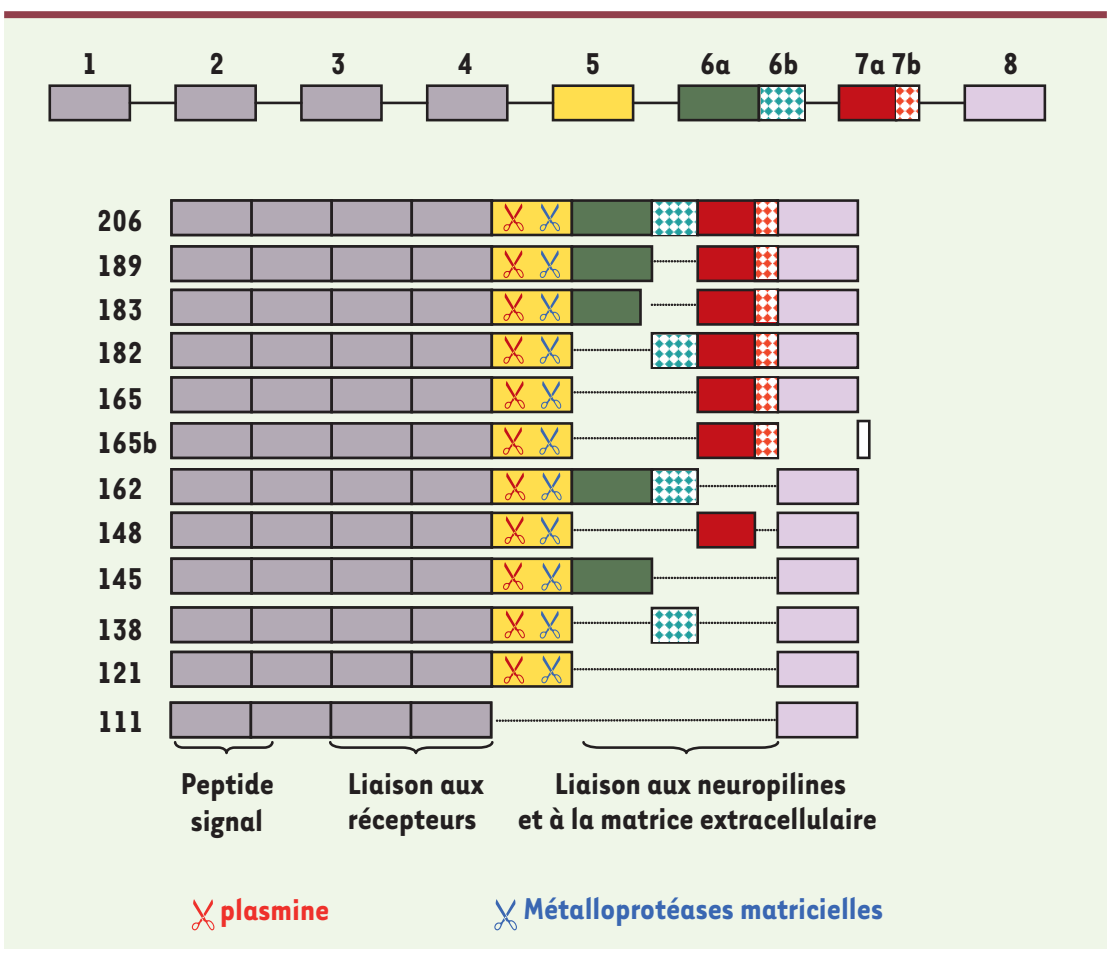

Figure 1. Variants du VEGF. Description de l'utilisation des exons dans les différents variants du $V \varepsilon G F$, et de la fonction des séquences qu'ils codent. Le numéro d'identification des variants correspond au nombre d'acides aminés contenus dans la molécule mature après excision du peptide signal, et la lettre b indique l'utilisation de l'exon 8b. Les exons ne sont pas dessinés à l'échelle.
I'injection intra-tumorale de camptothécine [4], suggère qu'il pourrait être exprimé par des patients traités par chimiothérapie. Si cette hypothèse est avérée, le VEGFlll pourrait progressivement devenir la forme majeure de VEGF en raison de la protéolyse souvent élevée dans l'environnement tumoral. Comme il ne semble pas indispensable à l'organisme, une thérapie ciblant spécifiquement le VEGFlll pourrait diminuer efficacement l'angiogenèse tumorale sans avoir les effets secondaires des traitements anti-VEGF actuels. Plusieurs stratégies sont envisageables: (1) inhibition de la liaison du VEGFlll aux récepteurs par des anticorps ou des aptamères spécifiques; (2) inhibition spécifique de l'expression de VEGFlll par ARN interférentiel; (3) inhibition du processus d'épissage impliqué dans la génération du VEGFl11; La description de molécules capables de corriger l'épissage aberrant de certains gènes sans entraver l'épissage constitutif [8, 9] laisse entrevoir de nouvelles solutions thérapeutiques. $\diamond$

VEGF111: Dr Jekyll and Mr Hyde?

\section{RÉFÉRENCES}

1. Buysschaert I, Carmeliet P, Dewerchin M. Clinical and fundamental aspects of angiogenesis and antiangiogenesis. Acta Clin Belg 2007 ; 62 : 162-9.

2. Keyt BA, Berleau LT, Nguyen HV, et al. The carboxylterminal domain (111-165) of vascular endothelial growth factor is critical for its mitogenic potency. J Biol Chem 1996; 271 : 7788-95.

3. Lee S, Jilani SM, Nikolova GV, et al. Processing of VEGF-A by matrix metalloproteinases regulates bioavailability and vascular patterning in tumors. J Cell Biol 2005 ; 169 : 681-91.

4. Mineur P, Colige AC, Deroanne CF, et al. Newly identified biologically active and proteolysisresistant VEGF-A isoform VEGFl11 is induced by genotoxic agents. J Cell Biol 2007 ; 179 : 1261-73.

5. Lauer G, Sollberg S, Cole M, et al. Expression and proteolysis of vascular endothelial growth factor is increased in chronic wounds. J Invest Dermatol 2000 ; $115: 12-8$.

6. Roth D, Piekarek M, Paulsson M, et al. Plasmin modulates vascular endothelial growth factor-Amediated angiogenesis during wound repair. Am J Pathol 2006; 168 : 670-84.

7. Folkman J. Tumor angiogenesis: therapeutic implications. N Engl J Med 1971 ; 285 : 1182-6.

8. Corcos L, Solier S. Épissage alternatif, pathologie et thérapeutique moléculaire. Med Sci (Paris) 2005 ; $21: 253-60$.

9. Soret J, Gabut M, Tazi J. SR proteins as potential targets for therapy. Prog Mol Subcell Biol 2006 ; 44 : 65-87. 